\title{
The First Law of Black Hole Mechanics
}

\author{
Robert M. Wald*
}

\begin{abstract}
A simple proof of a strengthened form of the first law of black hole mechanics is presented. The proof is based directly upon the Hamiltonian formulation of general relativity, and it shows that the the first law variational formula holds for arbitrary nonsingular, asymptotically flat perturbations of a stationary, axisymmetric black hole, not merely for perturbations to other stationary, axisymmetric black holes. As an application of this strengthened form of the first law, we prove that there cannot exist Einstein-Maxwell black holes whose ergoregion is disjoint from the horizon. This closes a gap in the black hole uniqueness theorems.
\end{abstract}

\section{Derivation of the First Law}

It was noted by Hilbert at the inception of general relativity that the Einstein field equations are derivable from an action principle,

$$
S=\frac{1}{16 \pi} \int R \sqrt{-g} d^{4} x
$$

Thus, general relativity has a Lagrangian formulation. The corresponding Hamiltonian formulation was given many years later in a collaboration between Charles Misner, Richard Arnowitt, and Stanley Deser. The main results of this collaboration are summarized in [1].

The Hamiltonian formulation of general relativity is employed as a starting point in all attempts to formulate a quantum theory of gravity via the canonical approach. It plays a less essential role within the context of purely classical general relativity. However, even in that context, the Hamiltonian formulation of general relativity provides some penetrating insights into the structure of the theory. In this paper, I shall illustrate this point by showing how a strengthened form of the first law

*Enrico Fermi Institute and Department of Physics, University of Chicago, 5640 S. Ellis Avenue, Chicago, IL 60637, USA. This research was supported in part by the National Science Foundation under Grant No. PHY89-18388. 
WALD : FIRST LAW

of black hole mechanics can be derived in a very simple and direct manner from the Hamiltonian formulation of general relativity. The results presented here were obtained in collaboration with D. Sudarsky and were first reported in [2]. I shall restrict attention here to Einstein-Maxwell theory-the more general case of EinsteinYang-Mills theory was considered in [2]-but the analysis generalizes straightforwardly to allow other fields, provided only that a Hamiltonian formulation of the complete theory can be given.

In the Hamiltonian formulation of Einstein-Maxwell theory, a point in phase space corresponds to the specification of the fields $\left(h_{a b}, \pi^{a b}, A_{a}, E^{a}\right)$ on a three dimensional manifold $\Sigma$. Here $h_{a b}$ is a Riemannian metric on $\Sigma$ and $A_{a}$ is the spatial part of the vector potential (i.e., the pull-back to $\Sigma$ of the spacetime vector potential $A_{\mu}$ ). The momentum canonically conjugate to $h_{a b}$ is $\frac{1}{16 \pi} \pi^{a b}$, where $\pi^{a b}$ is related to the extrinsic curvature, $K_{a b}$, of $\Sigma$ in the spacetime obtained by evolving this initial data by,

$$
\pi^{a b}=\sqrt{h}\left(K^{a b}-h^{a b} K\right)
$$

The momentum conjugate to $A_{a}$ is $\frac{1}{4 \pi} \sqrt{h} E^{a}$, where $E^{a}$ is the electric field in the evolved spacetime.

Constraints are present in Einstein-Maxwell theory. The allowed initial data is restricted to the constraint submanifold in phase space defined by the vanishing at each point $x \epsilon \Sigma$ of the following quantities,

$$
\begin{gathered}
0=\mathcal{C}=\frac{1}{4 \pi} \sqrt{h} D_{a} E^{a} \\
0=\mathcal{C}_{0}=\frac{1}{16 \pi} \sqrt{h}\left\{-R+2 E_{a} E^{a}+F_{a b} F^{a b}+\frac{1}{h}\left(\pi^{a b} \pi_{a b}-\frac{1}{2} \pi^{2}\right)\right\} \\
0=\mathcal{C}_{a}=-\frac{1}{8 \pi} \sqrt{h}\left\{D_{b}\left(\pi_{a}^{b} / \sqrt{h}\right)-2 F_{a b} E^{b}\right\}
\end{gathered}
$$

where $D_{a}$ is the derivative operator on $\Sigma$ compatible with $h_{a b}, R$ denotes the scalar curvature of $h_{a b}$, and $F_{a b}=2 D_{[a} A_{b]}$.

The ADM Hamiltonian, $H$, for Einstein-Maxwell theory has the "pure constraint" form,

$$
H=\int_{\Sigma}\left(N^{\mu} \mathcal{C}_{\mu}+N^{\mu} A_{\mu} \mathcal{C}\right)
$$

Here $N^{\mu}$ and $A_{0}$ are to be viewed as non-dynamical variables, which may be prescribed arbitrarily. In the spacetime obtained by solving Hamilton's equations, $N^{\mu}$ has the interpretation of being the time evolution vector field (i.e., its projection normal to $\Sigma$ yields the lapse function, $N$, and its projection into $\Sigma$ yields the shift vector, $N^{a}$ ), and $A_{0}$ has the interpretation of being the component of the vector potential normal to $\Sigma$. The "pure constraint" form of $H$ is not special to Einstein-Maxwell theory; any Hamiltonian arising from a diffeomorphism invariant theory always takes such a form (see the appendix of [3]). 
The derivation of the strengthened form of the first law of black hole mechanics is based upon the following three properties of the ADM Hamiltonian: (i) It vanishes identically on the constraint submanifold. Hence, its first order variation off of a solution vanishes whenever the varied initial data satisfies the linearized constraints. (ii) Its variation yields the Einstein-Maxwell equations. (iii) For suitable choices of time evolution vector field in an asymptotically flat spacetime, its variation is directly related to formulas for the variation of mass, charge, and angular momentum in the spacetime.

The first of these properties is manifest from eq. (6). The second property is just the statement of what we mean by $H$ being a Hamiltonian for Einstein-Maxwell theory. More explicitly, let $\left(h_{a b}, \pi^{a b}, A_{a}, E^{a}\right)$ be initial data (satisfying the constaints) for an Einstein-Maxwell solution, and let $\left(\delta h_{a b}, \delta \pi^{a b}, \delta A_{a}, \delta E^{a}\right)$ be an arbitrary perturbation (not necessarily satisfying the linearized constraints) of compact support on $\Sigma$. By integrating by parts, we can express the variation of $H$ in the form.

$$
\delta H=\int_{\Sigma}\left[P^{a b} \delta h_{a b}+\mathcal{Q}_{a b} \delta \pi^{a b}+R^{a} \delta A_{a}+S_{a} \delta\left(\sqrt{h} E^{a}\right)\right]
$$

Then, the coefficient, $P^{a b}$, of $\delta h_{a b}$ yields minus the "time derivative" (i.e., the Lie derivative with respect to $N^{\mu}$ ) of the canonical momentum $\frac{1}{16 \pi} \pi^{a b}$ in the solution to the Einstein-Maxwell equations arising from the initial data $\left(h_{a b}, \pi^{a b}, A_{a}, E^{a}\right)$. Similarly, the coefficient, $16 \pi \mathcal{Q}_{a b}$, of $\frac{1}{16 \pi} \delta \pi^{a b}$ yields the time derivative of $h_{a b}$, etc. (Explicit formulas for $P^{a b}, \mathcal{Q}_{a b}, R^{a}$, and $S_{a}$ are given in eqs. (19)-(23) of [2] in the more general case of Einstein-Yang-Mills theory.)

The third property can be understood and derived from the following considerations [4]: Let $\left(h_{a b}, \pi^{a b}, A_{a}, E^{a}\right)$ be initial data (satisfying the constaints) for an asymptotically flat spacetime. Suppose, now, that we consider variations $\left(\delta h_{a b}, \delta \pi^{a b}, \delta A_{a}, \delta E^{a}\right)$ of this initial data which are merely asymptotically flat (rather than being of compact support). Then extra terms will appear in eq. (7) due to contributions from boundary terms at infinity which arise when one does the integrations by parts needed to put the volume terms in the form (7). In the case where $N^{\mu}$ asymptotically approaches a time transition (i.e., the lapse function $N$ goes to 1 and the shift vector $N^{a}$ goes to 0 at infinity), one obtains (assuming that no other boundaries are present on $\Sigma$ - see below),

$$
\begin{gathered}
\delta H=\int_{\Sigma}\left[P^{a b} \delta h_{a b}+\mathcal{Q}_{a b} \delta \pi^{a b}+R^{a} \delta A_{a}+S_{a} \delta\left(\sqrt{h} E^{a}\right)\right] \\
-\frac{1}{16 \pi} \delta \oint_{\infty} d S^{a}\left[\partial^{b} h_{a b}-\partial_{a} h_{b}^{b}\right]-\frac{1}{4 \pi} \delta \oint_{\infty} d S^{a} A_{0} E_{a}
\end{gathered}
$$

Equation (8) suggests that we modify the definition of the ADM Hamiltonian by addition of a surface term,

$$
\tilde{H} \equiv H+\frac{1}{16 \pi} \oint_{\infty} d S^{a}\left[\partial^{b} h_{a b}-\partial_{a} h_{b}^{b}\right]+\frac{1}{4 \pi} \oint_{\infty} d S^{a} A_{0} E_{a}
$$


WALD : FIRST LAW

If we do so, then $\tilde{H}$ will act as a true Hamiltonian on phase space in the sense that its variation will be given by the right side of eq.(7) for all asymptotically flat perturbations. It is natural, then, to define the "canonical energy" $E$ on the constraint submanifold of phase space to be the numerical value of this true Hamiltonian. Hence, we obtain,

$$
\mathcal{E}=\frac{1}{16 \pi} \oint_{\infty} d S^{a}\left[\partial^{b} h_{a b}-\partial_{a} h_{b}^{b}\right]+\frac{1}{4 \pi} \oint_{\infty} d S^{a} A_{0} E_{a}=m+V Q
$$

where $m$ is the ADM mass, $V$ is the asymptotic value of $A_{0}$ at infinity, and $Q$ is the electric charge. (In the case presently considered - where there are no "boundaries" aside from infinity $-Q$ will vanish, but we keep this term in eq. (10) since it will be nonvanishing in the more general cases considered below.) In terms of the original ADM Hamiltonian $H$, we thereby obtain,

$$
\delta H=\int_{\Sigma}\left[P^{a b} \delta H_{a b}+\mathcal{Q}_{a b} \delta \pi^{a b}+R^{a} \delta A_{a}+S_{a} \delta\left(\sqrt{h} E^{a}\right)\right]-\delta m-V \delta Q
$$

which yields the desired relationship between the variation of $H$ and the variation of ADM mass, $m$, and charge, $Q$, in the case where $N^{\mu}$ approaches a time translation at infinity. In a similar manner, if $N^{\mu}$ asymptotically approaches a rotation at infinity, we obtain,

$$
\delta H=\int_{\Sigma}\left[P^{\prime a b} \delta h_{a b}+\mathcal{Q}_{a b}^{\prime} \delta \pi^{a b}+R^{\prime a} \delta A_{a}+S_{a}^{\prime} \delta\left(\sqrt{h} E^{a}\right)\right]+\delta J
$$

where $J$ is the "canonical angular momentum" defined by [2] (see also [5]),

$$
J=-\frac{1}{16 \pi} \oint_{\infty}\left(2 \phi_{b} \pi^{a b}+4 \phi^{b} A_{b} E^{a}\right) d S_{a}
$$

where $\phi^{a}$ is an asymptotic rotational Killing field on $\Sigma$. (In eq. (12), I have inserted primes on the quantities $P^{\prime a b}$, etc. appearing in the volume integral to alert the reader to the fact that these quantities depend upon the choice of $N^{\mu}$ and, hence are different in eqs. (11) and (12), since different choices of $N^{\mu}$ have been made. The Hamiltonian functions appearing on the left sides of these equations also, of course, are different for the same reason, but since I prefer to use $H$ to denote the Hamiltonian (6) for any choice of $N^{\mu}$, I have not inserted a prime on $H$ in eq. (12).) Equations (11) and (12) give explicit expression of property (iii) stated above.

The above formulas (11) and (12) are easily generalized to the case where $\Sigma$ is a manifold with boundary, i.e., when, in addition to having an asymptotically flat "end", $\Sigma$ also possesses a regular "interior boundary", $S$. In that case, the integrations by parts needed to put the "volume contribution" to the variation of $H$ in the form (7) also give rise to surface terms from $S$. These additional surface terms are readily computed (see [2] for their explicit form). 
WALD : FIRST LAW

The strengthened form of the first law of black hole mechanics follows directly from the above three properties of the Hamiltonian formulation of Einstein-Maxwell theory. Let $\left(M, g_{\mu \nu}, A_{\mu}\right)$ be a solution to the Einstein-Maxwell equations describing a stationary-axisymmetric black hole, whose event horizon is a bifurcate Killing horizon, with bifurcation surface $S$. Let $t^{\mu}$ and $\phi^{\mu}$ denote the Killing fields on this spacetime which, respectively, asymptotically approach a time translation and rotation at infinity. We assume that a Maxwell gauge choice has been made so that $A_{\mu}$ is nonsingular everywhere outside the black hole and on the event horizon, and satisfies $\mathcal{L}_{t} A_{\mu}=\mathcal{L}_{\phi} A_{\mu}=0$. (Note that the assumption that we can introduce a globally well defined vector potential restricts consideration to the case where the magnetic charge vanishes. However, there actually is no loss of generality in restricting attention to this case, since the magnetic charge always can be put to zero by means of a duality rotation.) Let

$$
\chi^{\mu}=t^{\mu}+\Omega \phi^{\mu}
$$

denote the linear combination of $t^{\mu}$ and $\phi^{\mu}$ which vanishes on $S$. (Equation (14) defines the "angular velocity of the horizon", $\Omega$.) Let $\Sigma$ be an asymptotically flat hypersurface which terminates on the bifurcation surface $S$. Let $\left(h_{a b}, \pi^{a b}, A_{a}, E^{a}\right)$ denote the initial data which is induced on $\Sigma$. Finally, let $H$ denote the ADM Hamiltonian asssociated with the time evolution vector field $N^{\mu}=\chi^{\mu}$.

Now, let $\left(\delta h_{a b}, \delta \pi^{a b}, \delta A_{a}, \delta E^{a}\right)$ denote any perturbation of the above initial data which is asymptotically flat, is nonsingular on $\Sigma$ (including $S$ ), and which satisfies the linearized constraint equations. Then, by property (i) above, we have $\delta H=0$ for this perturbation. However, by property (ii) above, together with the fact that $\chi^{\mu}$ is a symmetry of the background solution

(i.e., $\mathcal{L}_{\chi} g_{\mu \nu}=\mathcal{L}_{\chi} A_{\mu}=0$ ), it follows immediately that the "volume contribution" to $\delta H$ vanishes. Thus it is clear from eqs. (11) and (12) that property (iii) will give rise to a formula relating the variations in mass, angular momentum, and charge associated with the perturbation (i.e., the "surface terms" from infinity) to a surface contribution from $S$ (which was not included in eqs. (11) and (12) above). Since $N^{\mu}=\chi^{\mu}$ vanishes on $S$, the evaluation of this boundary term from $S$ simplifies considerably. The final result thereby obtained is the following [2]: For any nonsingular, asymptotically flat perturbation of a stationary, axisymmetric black hole with bifurcate horizon, we have,

$$
\delta m+V \delta Q-\Omega \delta J=\frac{1}{8 \pi} \kappa \delta A
$$

Here, $\kappa$ denotes the surface gravity (see, e.g., [6]) of the horizon and

$A$ denotes the area of $S$. (The term $\frac{1}{8 \pi} \kappa \delta A$ is, of course, just the surface contribution from $S$.) Equation (15) expresses the first law of black hole mechanics.

The above derivation of eq. (15) is considerably simpler than the original derivation given in [7]. More significantly, the result obtained here is considerably stronger: 
WALD : FIRST LAW

The derivation of [7] establishes that eq. (15) holds only for perturbations to other stationary, axisymmetric black holes. (An extension of the derivation of [7] to include a somewhat more general class of perturbations which are " $t=\phi$-symmetric" was given in [8].) The above derivation proves that eq. (15) holds for all nonsingular asymptotically flat perturbations which satisfy the linearized constraint equations. As we now shall show, this strengthened form of the first law will enable us to close a gap that had existed for many years in the proof of the black hole uniqueness theorems.

\section{Application to the Black Hole Uniqueness Theorems}

The conclusion that the charged Kerr solutions are the only stationary black hole solutions in Einstein-Maxwell theory rests on the combined work of many authors. One of the key steps in the argument leading to this conclusion is a theorem of Hawking [9], [10], which usually is quoted as asserting that a stationary black hole must either be static or axisymmetric. Under the assumption that the surface gravity, $\kappa$, is nonvanishing (corresponding to the case of a bifurcate Killing horizon - see [11]), Israel's theorem [12], [13] then proves that the only static black holes in Einstein-Maxwell theory are the Reissner-Nordstrom solutions (i.e., the charged Kerr solutions with vanishing angular momentum), whereas the combined work of Carter [14], Robinson [15], Mazur [16], and Bunting (see Carter [17]) establishes uniqueness of the charged Kerr solutions in the stationary, axisymmetric case.

However, Hawking's theorem actually states the following: First, the theorem asserts that the event horizon of a stationary black hole must be a Killing horizon, i.e., there must exist a Killing field $\chi^{\mu}$ in the spacetime which is normal to the horizon. If $\chi^{\mu}$ fails to coincide with the stationary Killing field $t^{\mu}$, then it is shown that the spacetime must be axisymmetric as well as stationary. In that case it follows immediately that eq. (14) will hold with $\Omega \neq 0$ - i.e., the black hole will be "rotating" -and $t^{\mu}$ will be spacelike in a neighborhood of the horizon, so that the black hole will be enclosed by an "ergoregion." On the other hand, if $t^{\mu}$ coincides with $\chi^{\mu}$ (so that the black hole is "non- rotating") AND if $t^{\mu}$ is globally timelike outside of the black hole (so that no "ergoregions" exist), then it is shown that the spacetime must be static. However, the case where $t^{\mu}$ coincides with $\chi^{\mu}$ but fails to be globally timelike outside of the black hole is not ruled out by the theorem, although plausibility arguments against this possibility have been given [10]; see also [18]. Consequently, the standard black hole uniqueness theorems leave open the following loophole: In principle, there could exist additional stationary black hole solutions to the Einstein-Maxwell equations with bifurcate horizon which are neither static nor axisymmetric. Such black holes would have to be nonrotating (in the sense that $t^{\mu}$ coincides with $\chi^{\mu}$ ) and also would have to have a nontrivial ergoregion. Furthermore, since $\chi^{\mu}$ automatically is timelike in a neighborhood of the horizon outside of the black hole (see [19]), this ergoregion would have to be disjoint from the horizon. I now shall show how this loophole can be 
closed by proving that any nonrotating black hole in Einstein-Maxwell theory whose ergoregion is disjoint from the horizon must be static-even if $t^{\mu}$ is not initially assumed to be globally timelike outside of the black hole. In particular, this gives a direct proof that there cannot exist black holes in Einstein-Maxwell theory whose ergoregion is disjoint from the horizon. The proof relies directly upon the strengthened form of first law of black hole mechanics obtained in the previous section, and thus provides an excellent example of the utility of this result.

Although the derivation of eq. (15) was given above for the case of a stationary, axisymmetric black hole, it is immediately clear that the derivation also applies for a black hole which is merely stationary (i.e., which possesses a Killing field $t^{\mu}$ which approaches a time translation near infinity) but is non-rotating in the sense that $t^{\mu}$ vanishes on $S$. In that case, we obtain,

$$
\delta m+V \delta Q=\frac{1}{8 \pi} \kappa \delta A
$$

i.e., eq. (15) holds with $\Omega=0$. Hence, as an immediate corollary of our strengthened form of the first law of black hole mechanics, we obtain the following result: For an arbitrary stationary, nonrotating Einstein-Maxwell black hole, any nonsingular, asymptotically flat perturbation of the initial data which satisfies the linearized constraint equations, preserves the charge, $\mathbf{Q}$, of the black hole and preserves the area, $\mathbf{A}$, of $\mathbf{S}$ cannot result in a first order change the ADM mass, $\mathbf{m}$, of the spacetime.

We now shall attempt to explicitly construct a perturbation which violates this corollary. As we shall see, this attempt will succeed unless the spacetime is static. Consequently, we shall conclude that every stationary, non-rotating Einstein-Maxwell black hole must be static.

The first (and, technically, most difficult) step in the argument is to prove that in the (unperturbed) stationary black hole spacetime, a maximal (i.e., vanishing trace extrinsic curvature) slice, $\Sigma$, always can be chosen which intersects the bifurcation surface, $S$, is asymptotically flat and is asymptotically orthogonal to $t^{\mu}$ at infinity. A proof that such a slice exists is given in [20], and we refer the reader to that reference for further details.

Now, let $\left(h_{a b}, \pi^{a b}, A_{a}, E^{a}\right)$ be the initial data which is induced on the maximal slice, $\Sigma$, of the previous paragraph for an (unperturbed) nonrotating Einstein-Maxwell black hole. Consider the following perturbation of this initial data:

$$
\begin{gathered}
\delta h_{a b}=4 \phi h_{a b} \\
\delta \pi^{a b}=-4 \phi \pi^{a b}-\pi^{a b} \\
\delta A_{a}=-A_{a} \\
\delta E^{a}=-6 \phi E^{a}
\end{gathered}
$$


WALD : FIRST LAW

where $\phi$ is the solution to

$$
D^{a} D_{a} \phi-\mu \phi=\rho
$$

on $\Sigma$ determined by the boundary conditions $\phi \rightarrow 0$ at infinity and $\phi=0$ on $S$, where

$$
\begin{gathered}
\mu=\frac{1}{h} \pi^{a b} \pi_{a b}+E^{a} E_{a}+\frac{1}{2} F_{a b} F^{a b} \\
\rho=\frac{1}{4}\left[\frac{1}{h} \pi^{a b} \pi_{a b}+F_{a b} F^{a b}\right]
\end{gathered}
$$

Then it may be verified directly that this perturbation satisfies the linearized constraint equations and also satisfies $\delta Q=\delta A=0$. However, it also can be proven [2] that this perturbation satisfies $\delta m<0$ unless $\rho=0$. Consequently, a contradiction with the first law of black hole mechanics will be obtained unless $\pi^{a b}=0$ (and also $F_{a b}=0$ ), i.e., the first law implies that the full extrinsic curvature of $\Sigma$ must vanish. By isometry invariance, the one-parameter family of slices, $\Sigma_{t}$, obtained by "time translating" $\Sigma$ along the orbits of $t^{\mu}$ also must have vanishing extrinsic curvature. However, it then follows that the projection of $t^{\mu}$ normal to these hypersurfaces (i.e., $t^{\prime \mu}=-\left(t^{\nu} n_{\nu}\right) n^{\mu}$, where $n^{\mu}$ is the unit normal field to $\left.\Sigma_{t}\right)$ must be a Killing field. (Indeed, since $t^{\prime} \mu$ approaches $t^{\mu}$ at infinity, we actually must have $t^{\prime} \mu=t^{\mu}$.) Hence, the nonrotating black hole possesses a hypersurface orthogonal, timelike Killing field which is everywhere timelike outside the black hole and approaches a time translation at infinity. Thus, the black hole is static, as we desired to show.

Interestingly, this proof does not generalize to Einstein-Yang-Mills case. Indeed, it is argued in [2] that nonrotating black holes which fail to be static will occur in Einstein-Yang-Mills theory, although such solutions, if they exist, should be unstable.

\section{References}

[1] R. Arnowitt, S. Deser, and C.W. Misner, "The Dynamics of General Relativity", in Gravitation: An Introduction to Current Research, ed. L. Witten (Wiley, New York, 1962).

[2] D. Sudarsky and R.M. Wald, Phys. Rev. D 46, 1453 (1990).

[3] J. Lee and R.M. Wald, J. Math. Phys. 31, 725 (1990).

[4] T. Regge and C. Teitelboim, Ann. Phys. 88, 286 (1974).

[5] J.D. Brown, E.A. Martinez, and J.W. York, Jr., Phys. Rev. Lett. 66, 2281 (1991).

[6] R.M. Wald, General Relativity (University of Chicago Press, Chicago, 1984).

[7] J.M. Bardeen, B. Carter, and S.W. Hawking, Commun. Math. Phys. 31, 161 $(1973)$. 
WALD : FIRST LAW

[8] S.W. Hawking, Commun. Math. Phys. 33, 323 (1973).

[9] S.W. Hawking, Commun. Math. Phys. 25, 152 (1972).

[10] S.W. Hawking and G.F.R. Ellis, The Large Scale Structure of Spacetime (Cambridge University Press, Cambridge, 1973).

[11] I. Racz and R.M. Wald, Class. and Quant. Grav. 9, 2643 (1992).

[12] W. Israel, Phys. Rev. 164, 1776 (1967).

[13] W. Israel, Commun. Math. Phys. 8, 245 (1968).

[14] B. Carter, in Black Holes, ed. by C. DeWitt and B.S. DeWitt (Gordon and Breach, New York, 1973).

[15] D.C. Robinson, Phys. Rev. Lett. 34, 905 (1975).

[16] P.O. Mazur, J. Phys. A15, 3173 (1982).

[17] B. Carter, Commun. Math. Phys. 99, 563 (1985).

[18] P. Hajicek, Phys. Rev. D7, 2311 (1973).

[19] B.S. Kay and R.M. Wald, Phys. Rep. 207, 49 (1991).

[20] P. Chrusciel and R.M. Wald, "Maximal Hypersurfaces in Aymptotically Stationary Spacetimes", to be published. See also, R. M. Wald, "Maximal Slices in Stationary Spacetimes with Ergoregions" in Brill Festschrift (Vol. 2 of this Proceeding), ed. B. L. Hu and T. A. Jacobson 